\title{
L'influence de la fratrie sur la socialisation des enfants âgés de 24 à 36 mois et gardés en crèche collective
}

\author{
Johanna Trichet
}

Educatrice de jeunes enfants et étudiante en master de psychologie (université Toulouse-Jean-Jaurès)

Anaïs Cardon

Étudiante en master de psychologie (université Toulouse Jean-Jaurès)

Olivia Troupel

Docteure et maître de conférences en psychologie du développement

(université Toulouse-Jean-Jaurès-UMR- 5193- lisst-CERS)

Grâce aux 22 crèches de Haute-Garonne participantes et au volontariat des parents sollicités, 125 enfants ont participé à cette recherche afin de déterminer l'impact de la fratrie sur la socialisation des jeunes enfants accueillis en crèche collective. L'âge des participants varie de 24 à 36 mois, avec une moyenne d'âge de 30 mois. Parmi ces enfants, 53,6\% sont issus d'une fratrie. L'échantillon est composé de 76 filles et de 49 garçons.

Les professionnelles référentes des 125 enfants cibles ont complété le Profil SocioAffectif (PSA de La Freniere, Dubeau, Capuano, \& Janosz, 1990). Ce questionnaire a été rempli par les professionnels des crèches dans lesquelles les enfants sont accueillis au quotidien, afin de recueillir des informations relatives aux comportements de chaque enfant en collectivité. L'outil s'intéresse aux compétences sociales des enfants ainsi qu'à leurs difficultés d'adaptation au milieu. Le questionnaire se compose de huit échelles de base comportant chacune dix énoncés, dont la moitié concerne les difficultés d'adaptation, et l'autre moitié s'intéresse aux compétences sociales. Les trois premières échelles de base rendent compte des caractéristiques affectives de l'enfant. Les trois échelles suivantes évaluent plus particulièrement les interactions sociales avec les pairs. Enfin, les deux dernières concernent les relations de l'enfant avec les adultes. 
Trichet, J., Cardon, A., Troupel, O. \& Zaouche Gaudron, C. (2017). L'influence de la fratrie sur la socialisation des enfants âgés de 24 à 36 mois et gardés en crèche collective. Spirale, 81

À partir de ces huit échelles de base, il est possible d'obtenir les quatre échelles globales suivantes :

1. «Compétences sociales », afin de mesurer la maturité affective, la flexibilité et l'adaptation positive de l'enfant ;

2. «Problèmes intériorisés », qui rend compte des difficultés affectives vécues par l'enfant ;

3. «Problèmes extériorisés » pour évaluer les difficultés comportementales de l'enfant ;

4. «Adaptation générale » fournit un indice global du développement socio-affectif de l'enfant au sein du milieu dans lequel il est évalué.

Parallèlement, un second questionnaire, renseigné par les parents, a été créé pour les besoins de la recherche afin d'appréhender les habitudes et contextes de vie de chacun des enfants.

Cette recherche n'a pas permis de démontrer l'influence de l'appartenance à une fratrie sur le développement social précoce de l'enfant entre 24 et 36 mois.

En effet, les enfants uniques se sont montrés aussi adaptés à la collectivité et compétents socialement que les enfants cadets. Ils ne semblent pas rencontrer plus de difficultés affectives et comportementales au sein de la crèche, ne se sentant pas plus insécurisés dans cet environnement collectif. Ils ont fait preuve des mêmes capacités de gestion des émotions négatives et de la frustration que les enfants cadets, et semblent vivre aussi bien le cadre et les limites posés par l'adulte.

Dans la relation aux pairs, les enfants uniques et les enfants cadets se sont montrés tout autant capables d'empathie et de sensibilité envers autrui. Ils ont également fait preuve d'un niveau équivalent d'autonomie. En revanche, les enfants uniques semblent mieux intégrés au groupe de pairs et sont plus participatifs. La généralisation de l'accueil précoce en collectivité dans notre pays semble offrir aux enfants uniques des possibilités similaires de développement, ce qui pourrait expliquer cette absence de différence entre enfant cadet et enfant unique.

Nous avons alors souhaité savoir si d'autres facteurs avaient une influence sur les comportements sociaux de l'enfant au sein de la crèche. Ainsi, la catégorie socioprofessionnelle des parents semble avoir un impact ; les enfants avec des pères sans emploi au moment de la passation ont démontré de meilleures capacités d'adaptation sociale que les autres. Ces enfants 
Trichet, J., Cardon, A., Troupel, O. \& Zaouche Gaudron, C. (2017). L'influence de la fratrie sur la socialisation des enfants âgés de 24 à 36 mois et gardés en crèche collective. Spirale, 81

ne manifestent que peu d'anxiété. Ces résultats pourraient s'expliquer par une disponibilité accrue du père et un temps de présence auprès de l'enfant plus important au quotidien.

Par ailleurs, les enfants ayant intégré plus tardivement la crèche sont ceux qui montrent la meilleure adaptation à cet environnement. Ils sont plus compétents, sur les versants de la coopération et du contrôle des émotions négatives, que les enfants entrés avant 11 mois. Les enfants accueillis à temps complet semblent mieux intégrés au sein du groupe de pairs et font preuve de plus d'habiletés à s'adapter aux difficultés que les enfants accueillis à temps partiel. De plus, les enfants ayant un référent au sein de la crèche coopèrent mieux avec l'adulte, respectent mieux les règles et sont plus autonomes. Néanmoins, les enfants qui n'ont pas de référent montrent moins de difficultés affectives, et sont plus participatifs et plus confiants.

Le fait de fréquenter d'autres enfants que les pairs de la crèche ne semble pas influencer les compétences sociales de l'enfant entre 24 et 36 mois. Cependant, les enfants qui en fréquentent d'autres dans plusieurs contextes différents réagissent de manière plus adaptée face aux difficultés. Ainsi, la pluralité des contextes et des rencontres impacterait positivement les capacités d'adaptation du jeune enfant à son milieu.

\section{Bibliographie}

P.J. La Freniere, D. Dubeau, F. Capuano, M. Janosz, (1990). Profils socioaffectifs des enfants d'âge préscolaire, Revue canadienne de psychoéducation, 19 (1), p. 23-41. 